\title{
Editorial
}

\section{Gestión del conocimiento en Medicina Familiar, el caso de Ciudad Obregón, Sonora, México}

\author{
Management of Knowledge in Family Medicine, the Case of Ciudad Obregon, Sonora, Mexico \\ Gestão do Conhecimento em Medicina de Família $O$ caso de Ciudad Obregon, Sonora, México
}

\author{
Miguel Jiménez-Llamas*
}

A la par que evoluciona la especie humana, aparecen nuevas enfermedades y nuevos problemas que afectan a las familias; las situaciones que les aquejan -de índole social, económico, biológico o psicológicorepercuten en su estado de salud, ya sea en uno de sus integrantes o bien, en toda la familia.

Al ser la familia la unidad básica de la sociedad, debe ser de interés, no solo para los sistemas de salud, sino para las estructuras gubernamentales a fin de mantener un estado de bienestar dado el importante rol que ésta desempeña en el desarrollo de una comunidad y de una nación.

Por su parte, la medicina también evoluciona y desde la década de 1970, se inició en México el estudio y práctica de la Medicina Familiar como la disciplina académica que, mediante un cuerpo de conocimientos propios, se ha especializado en la atención de la familia, de la persona, del ente humano, y por tanto, de la salud de la población, denominada hoy como "atención primaria a la salud". Dentro de esta última se consideran los problemas sociales, que en muchos casos obedecen a condiciones socioeconómicas inestables, lo que constituye a la Medicina Familiar una prioridad para el sistema de salud. De manera estratégica, esta especialidad formula un espacio para ampliar el objeto de análisis, para abordarlo integral y holísticamente, al actuar antes de la enfermedad, en el diagnóstico temprano, el tratamiento oportuno y la prevención de complicaciones.

Actualmente, el perfil del médico familiar, debe estar definido, no solo por características asistenciales o de atención clínica, sino también por conocimientos metodológicos, estadísticos e informáticos, así como actitudinales, que le lleven a evaluar objetivamente los resultados de sus investigaciones para aplicarlos en la práctica médica. Es preciso contribuir a la creación de conocimientos que se transformen en prácticas compartidas entre los integrantes de una unidad médica, resultado de evidencias científicas que a la vez, ayuden en la toma de decisiones de los cuerpos directivos para alcanzar sus objetivos y/o metas, y cuyo resultado sea el obtener ventajas competitivas que sean sostenibles en el tiempo.

La investigación científica se convierte en una herramienta que contribuye poderosamente a la gestión del conocimiento, para lo cual, las unidades de Medicina Familiar cuentan con la estructura y recursos para desarrollar prácticas exitosas que cooperen con la toma de decisiones asertivas para el logro de los objetivos y metas trazados por la organización.

De esta manera, la difusión de conocimientos a través de los siguientes artículos, coadyuvará a compartir el conocimiento para hacerlo explícito y cerrar un ciclo en este proceso de gestión.

Felicito e invito a que los médicos familiares -como pilares del sistema de seguridad social que representa el Instituto Mexicano del Seguro Social- tanto en el estado de Sonora, como en todas las entidades del país, continúen generando ideas que innoven y mejoren la prestación de servicios de atención a la salud, tanto de manera preventiva como curativa, incidiendo corresponsablemente en la familia, en su bienestar y en su funcionalidad.

Este artículo debe citarse: Jiménez-Llamas M. Gestión del conocimiento en Medicina Familiar, el caso *Delegado estatal del Instituto de Ciudad Obregón, Sonora, México. Aten Fam. Mexicano del Seguro Social 2015;22(3):63. 